\title{
Digitalisierung der Engineering Prozesse durch System Lifecycle Management (SysLM)
}

Martin Eigner

Dieser Beitrag erläutert die Umsetzung der Digitalisierung speziell im Anwendungsgebiet des Engineering. Dabei wird die Digitalisierung, sowohl des eigentlichen Produktes als auch der im Rahmen von Service-orientierten Geschäftsmodellen entwickelten Dienstleistung, vorgestellt. Ein weiterer wesentlicher Punkt der Digitalisierung sind die horizontale und vertikale Integration der technischen und administrativen Arbeitsprozesse entlang des Produktlebenszyklus. Bei den vertikalen Integrationen handelt es ich um die Integrationen der Autorensysteme entlang den Lebenszyklusphasen Anforderungsmanagement, Systemarchitektur, CAD in Mechanik, Elektronik, Softwareentwicklung, Simulation, Test, Prozessplanung und Digitale Fabrik. Bei der horizontalen Integration stehen die administrativen Funktionen wie Freigabe-, Änderungs- und Konfigurationsmanagement über den gesamten Produklebenszyklus und die technische Integration der in den einzelnen Produktlebenszyklus-Phasen erzeugten Informationen im Vordergrund. Hier ist vor allem die Verteilung und Orchestrierung der Funktionen zwischen SysLM, MES und PPS relevant.

Keywords: Digitalisierung des Engineering, Engineering 4.0, System Lifecycle Management, Model Based Systems Engineering, System Thinking, IOT/IOS

\section{Digitalisierung des Engineerings - Grundlagen}

Digitalisierung, ist das schon wieder ein neues Buzzword? Ältere Leser kennen vielleicht noch den Begriff CIM (Computer Integrated Manufacturing) aus den 80er Jahren? "CIM beschreibt den integrierten EDV-Einsatz in allen mit der Produktion zusammenhängenden Betriebsbereichen. Es umfasst das informationstechnische Zusammenwirken zwischen CAD, CAP, CAM, CAQ und PPS. Hierbei soll die Integration der technischen und organisatorischen Funktionen zur Produkterstellung erreicht werden" (Definition laut AWF (Ausschuss für wirtschaftliche Fertigung, 1985). Einige erinnern sich vielleicht noch, der Begriff verschwand genauso schnell aus der öffentlichen Diskussion wie er aufkam. Aus unterschiedlichsten Gründen stieß diese Vision an Grenzen, so dass viele Experten sie als gescheitert angesehen haben. War die Zeit noch nicht reif, waren das Management überfordert oder gab es noch nicht die richtigen IT-Werkzeuge? Nichtsdestotrotz bei genauer Auslegung der Definition, handelte es sich bereits um eine Teildigitalisierung der Prozesskette Produktentwicklung zu Produktionsplanung und Produktion. Nach der kurzen CIM Euphorie und der Lean-Welle gewinnt mit 
Industrie 4.0, IOT und IOS die Digitalisierung und Automatisierung der Unternehmensprozesse auf einer neuen Evolutionsstufe wieder eine starke Bedeutung. Signifikant verbesserte methodische Ansätze und leistungsfähige IT-Werkzeuge rücken die Vision von CIM 35 Jahre später plötzlich in greifbare Nähe. Die Dimension der Digitalisierung ist jedoch ungleich größer und disruptiver. Sie beeinflusst jede Branche, den gesamten Wertschöpfungsprozess von den Anforderungen über verschiedenen Entwicklungsphasen, Simulation, Produktionsplanung und Produktion den operativen Betrieb bis zum Recycling. Sie bezieht sich auf innovative Produkte, Systeme und Dienstleistungen, sowie auf alle Geschäftsprozesse, Kunden, Dienstleister und Lieferanten. Das Internet der Dinge und Services (IOT/IOS) (Ashton, 2009) sowie Industrie 4.0 gehen in der Zukunft von vernetzten Produkten, Systemen und Dienstleistungen aus. Der Anteil an Elektronik und Software wird bei dieser Art von Produkten und eingebetteten Dienstleistungen kontinuierlich steigen. Kommunizieren Produkte miteinander über das Internet, wird von Cyberphysischen (Gill, 2009; Broy, 2010) bzw. Cybertronischen Systemen (Eigner et al. 2017) gesprochen. Die Entwicklung dieser neuen Systeme wird mehrere Konsequenzen nach sich ziehen: interdisziplinäre, regional und organisatorisch verteilte sowie integrierte Produktentwicklung, ein Überdenken heutiger Konstruktionsmethoden, Prozesse, IT-Lösungen und Organisationsformen sowie die Forderung nach durchgängigen Prozessketten, basierend auf digitalen Modellen in der Anforderungsdefinition, Systemarchitektur, Produktentwicklung, Simulation, Produktionsplanung, Produktion und Service. Es wird ein moderner, interdisziplinärer und systemorientierter Produkt Engineering Prozess (PEP) benötigt.

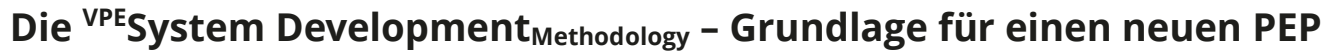

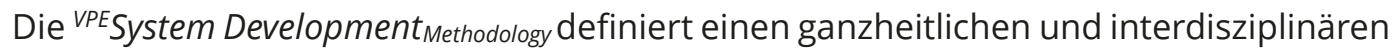
PEP (Die Entwicklungsmethodik wurde auf der EEE 2019 vorgestellt, Eigner et al, 2019; Eigner 2021). Sie übernimmt und fusioniert Konzepte unterschiedlicher interdisziplinärer und disziplinspezifischer Entwicklungs-Methodiken und passt sie an die Anforderungen und Randbedingungen der Cybertronik an (Eigner et al, 2017). Basierend auf den Definitionen einer grundlegenden Methodik nach Martin (1997) und Estefan (2008) besteht die Methodik aus drei wesentlichen Komponenten (vgl. Abbildung 1):

- Das Model Based Virtual Product Engineering (MVPE) Prozessmodell ist an den Kontext des Internets der Dinge und des interdisziplinären Designs angepasst.

- Das Kaiserslautern System Concretization Model (KSCM) ist als entsprechende Design-Methode positioniert

- Auf der Toolebene wird eine 5-Level-IT-Architekturkonzept auf Basis eines Engineering Backbone vorgeschlagen (Erweitert nach dem 4-Level VDA Konzept) 


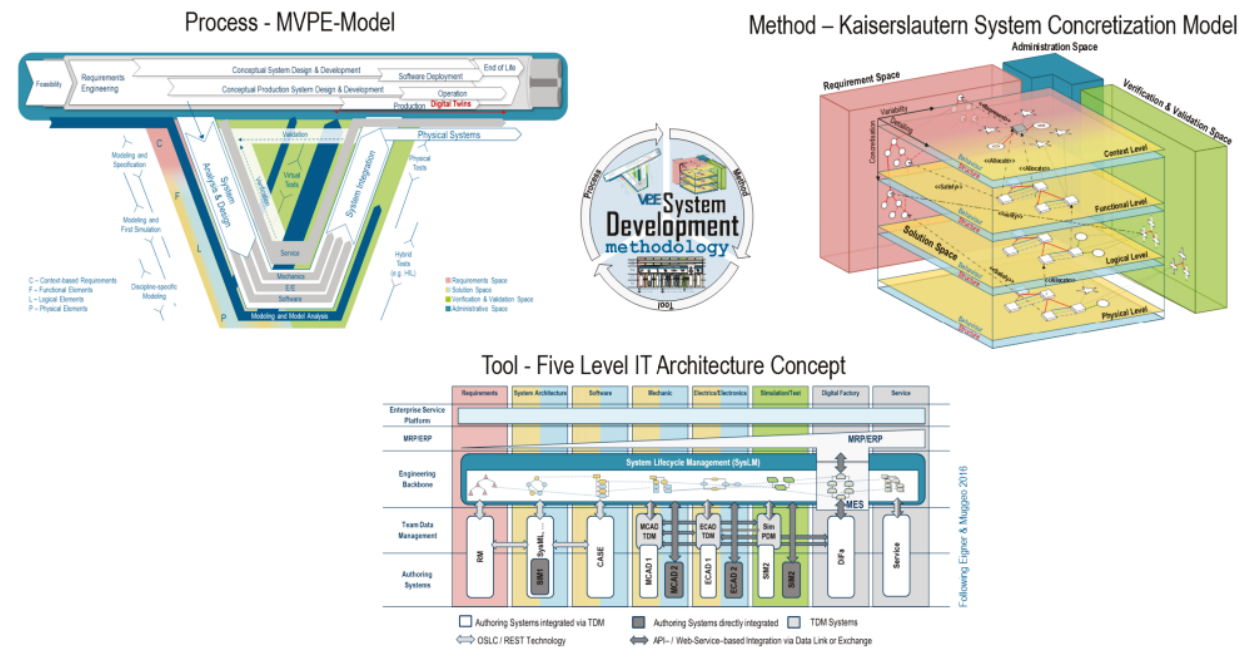

Abbildung 1: VPESystem Development Methodology

(Eigner et al., 2019; Eigner, 2021)

Während das MVPE-Modell - mit einem spezifischen Fokus auf konzeptionelle Gestaltung und Entwicklung - die allgemeinen Lebenszyklusphasen eines Produkt- oder Systementwicklungsprozesses beschreibt, bündelt das KSCM Techniken, um die Aufgaben zu erfüllen, die sich in den Phasen der konzeptionellen Gestaltung und disziplinspezifischen Entwicklung (-> Methode) ergeben. Das 5-Stufige IT-Architekturkonzept beschreibt einen komplementären Ansatz für die werkzeugbasierte Erstellung und Verwaltung von Systemdaten über den gesamten Lebenszyklus und die Umsetzung der horizontalen und vertikalen Integration.

\section{Vom Silo-orientierten Denken zum interdisziplinären Systemansatz}

Die wichtigsten Veränderungen in der Produkt- und Prozesswelt sind einerseits völlig neue Anforderungen an Produkte und Produktionssysteme und serviceorientierte Geschäftsmodelle sowie neue IT-Technologien, Architekturen und Standards (OSLC/REST/RDF, Web-Service, Micro-Services, multi-tenant-Datenbanken, Saas-Cloud), die die Umsetzung dieser Anforderungen ermöglichen. Die Digitalisierung von Produkten und Produktentwicklung bedeutet einen Transformationsprozess, der die klassischen Grenzen einer fragmentierten und konkurrierenden IT-Lösungswelt neu ordnet. Ein leichtgewichtiger und föderierter Engineering-Backbone wird die Rolle der Datenund Prozessintegration zwischen Produkt- und Produktionsentwicklung, Produktion/Herstellung und Montage sowie Service spielen. In Erweiterung der traditionellen PLM Anwendung wird diese zentrale Engineering Backbone-Lösung System Lifecycle 
Management (SysLM) genannt (Eigner, 2014, 2021). SysLM erweitert den PLM-Ansatz upstream (vollständige Integration von Systemmodellierung) und downstream (Integration von Engineering und Produktionsplanung) und basiert auf moderner WEBbasierender Software-Technologie. Für die mentale Transformation siloorientierten Denkens nach einem interdisziplinären und systemtechnischen Engineering Ansatz und die IT-technische Umsetzung von Engineering 4.0, können Model Based Systems Engineering (MBSE), System Thinking (ST), Advanced System Engineering (ASE) und SysLM als Leitlinie dienen.

\section{Umsetzung der Digitalisierung des Engineerings}

Dieses Kapitel erläutert die Umsetzung der im vorherigen Kapitel vorgestellten Trends und Methodiken der Digitalisierung speziell im Anwendungsgebietes des Engineerings. Dabei wird die Digitalisierung sowohl des Produktes als auch der im Rahmen von Service-orientierten Geschäftsmodellen entwickelten Dienstleistungsprodukte vorgestellt. Ein weiterer wesentlicher Punkt der Digitalisierung sind die horizontale und vertikale Integration der technischen und administrativen Engineering Prozesse entlang des Produktlebenszyklus (Abbildung 2). Bei den vertikalen Integrationen handelt es ich um die Integrationen der Autorensysteme entlang den Lebenszyklusphasen Anforderungsmanagement, Systemarchitektur, CAD in Mechanik, Elektronik und CASE und Simulation. Bei der horizontalen Integration stehen die administrativen Funktionen wie Freigabe-, Änderungs- und Konfigurationsmanagement über den gesamten Produklebenszyklus und die technische Integration der in den einzelnen Phasen erzeugten Informationen im Vordergrund.

Die Digitalisierungsstrategie muss neben den Produkt- und Prozess-relevanten Kriterien die agierenden und beteiligten Menschen, die Firmenkultur, die Organisationsstruktur und die aktuellen Technologien einbeziehen.

Wie im ersten Kapitel beschrieben, geht IOT von vernetzten und miteinander kommunizierenden Systemen und darauf aufbauenden Dienstleistungen aus ( $\rightarrow$ IOS). Durch gegenseitige Vernetzung und Beeinflussung wird der Funktionsumfang aktueller mechatronischer Systeme signifikant erweitert. Kommunizieren diese - i.d.R. über das Internet - miteinander, wird von Cyberphysischen Systemen (CPS) bzw. Cybertronischen Systemen (CTS) gesprochen. Beide stellen eine Weiterentwicklung mechatronischer Systeme in Richtung Intelligenz und Kommunikationsfähigkeit dar. Ein einfaches Beispiel, dass die Möglichkeiten eines Cybertronischen Produkts aufzeigt, ist die bekannte Kontaktlinse von Google (Abbildung 3). Diese intelligente Art von Produkten nennt man auch Smart Product. 


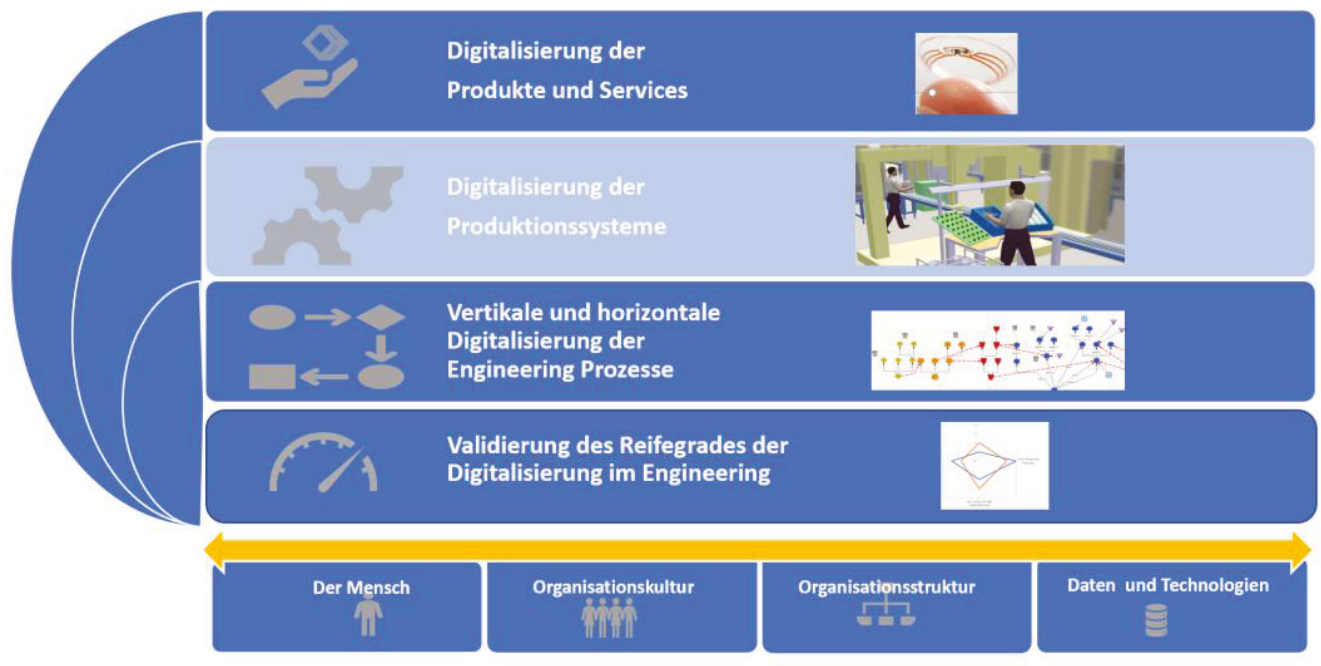

Abbildung 2: Die Digitalisierung des Engineerings / Engineering 4.0 (Eigner, 2021)

\section{Die Digitalisierung der Produkte und Services}

Auf der Basis kommunizierender ,Dinge' ( $\rightarrow$ Produkte/Systeme) werden neue, oftmals disruptive dienstleistungsorientierte Geschäftsmodelle für verschiedene Anwendungen aufgebaut, z.B. Smart Products, Smart Factory, Smart Energy, Smart Mobility, Smart Farming und Smart Buildings. Dienstleistungen innerhalb neuer Gesamtsysteme werden zum zentralen Erfolgsfaktor des Internet of Service (IOS). Sie können in offenen Netzen mit anderen Produktsystemen kommunizieren und kooperieren, und vernetzen sich so zu intelligenten, teils autonomen, sich selbst anpassenden Full-Service Systemen. Abbildung 4 zeigt ein Forschungskonzept einer Optimierung der Fahrwege eines Müllwagens durch Füllstandsmessung der Mülltonnen.

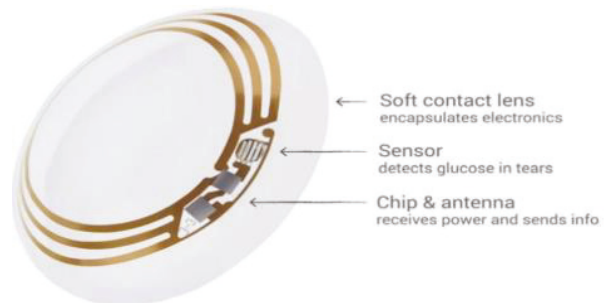

Abbildung 3: Die intelligente Kontaktlinse zur Blutzuckermessung (Quelle: Google) 


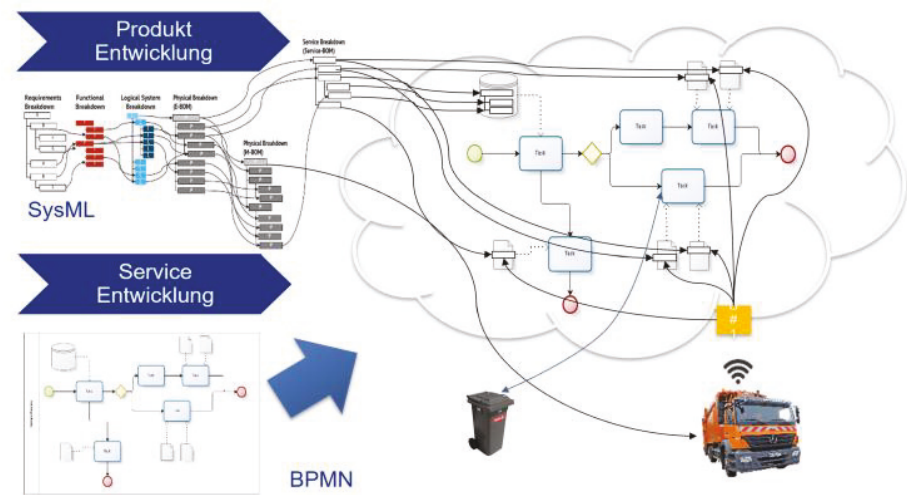

Abbildung 4: Full Service System am Beispiel Müll 4.0 (Quelle: VPE, M. Menges)

\section{Die Digitalisierung der Engineering Prozesse}

Neben der Digitalisierung von Produkten, Systemen und Services steht auch eine Digitalisierung der Engineering Prozesse in der Roadmap einer Digitalisierungsstrategie. Die Integration der etablierten Technologie des Product Lifecycle Managements (PLM) und der neueren Technologie des Model-Based Systems Engineerings (MBSE) wurde in Ihrer Relevanz für die Umsetzung von Industrie 4.0 erkannt. So heißt es: „Zentrale Themen sind hier das PLM-gestützte Engineering, welches das Produkt- und Produktionsdesign verbindet und eine durchgängige Unterstützung über die gesamte Wertschöpfung hinweg ermöglicht. Dies adressiert fachliche Punkte wie die integrierte Betrachtung von Systems Engineering, Modellierung und Simulation“ (Plattform Industrie 4.0, https://www.plattform-i40.de/PI40/Navigation/DE/Home/home.html, zugegriffen 7.3.2021). Unter Digitalisierung der Engineering Prozesse soll im Folgenden nicht nur die Existenz von Lebenszyklus-spezifischen IT-Lösungen, sondern auch die Integration, d.h. die modellbasierende Übergabe, von Informationen über IT-Systeme hinweg verstanden werden. Dieser Einsatz von Modellen anstatt von Dokumenten ist vorteilhaft in Entwicklungsszenarien, in welchen mehrere Disziplinen involviert sind und auf Grund von internationalen Normen Nachverfolgbarkeit der Produkte gefordert wird.

Unter vertikaler Integration soll hier entsprechend Abbildung 5 die Integration von Autorensystemen direkt oder über eine TDM Lösungen zu einem SysLM Backbone und umgekehrt vorgestellt werden. Gerade die ,Up Stream“ Potentiale, also die Systemmodellierung und die darin integrierte Anforderungsmodellierung inklusive der Anbindung an die administrative Ebene $(\rightarrow$ SysLM) sind heute noch wenig industriell eingesetzt. Die Prozesse sind noch nicht voll ausgereift. Aber die Projekte in diesem Bereich 
steigen stetig. Die Relevanz dieser Integration in der frühen Phase des Produktlebenszyklus wird von Experten allgemein anerkannt und wird sich in den nächsten Jahren zunächst in den Bereichen High Tech, Automotive, Medical, Aerospace und Defense zunehmend durchsetzen. Dagegen ist die Integration von M-CAD und E-CAD Systemen in TDM und PLM/SysLM seit vielen Jahren Standard. Heute eher noch wenig eingesetzten Bereiche der Integration sind die Software Entwicklung und die Simulation.

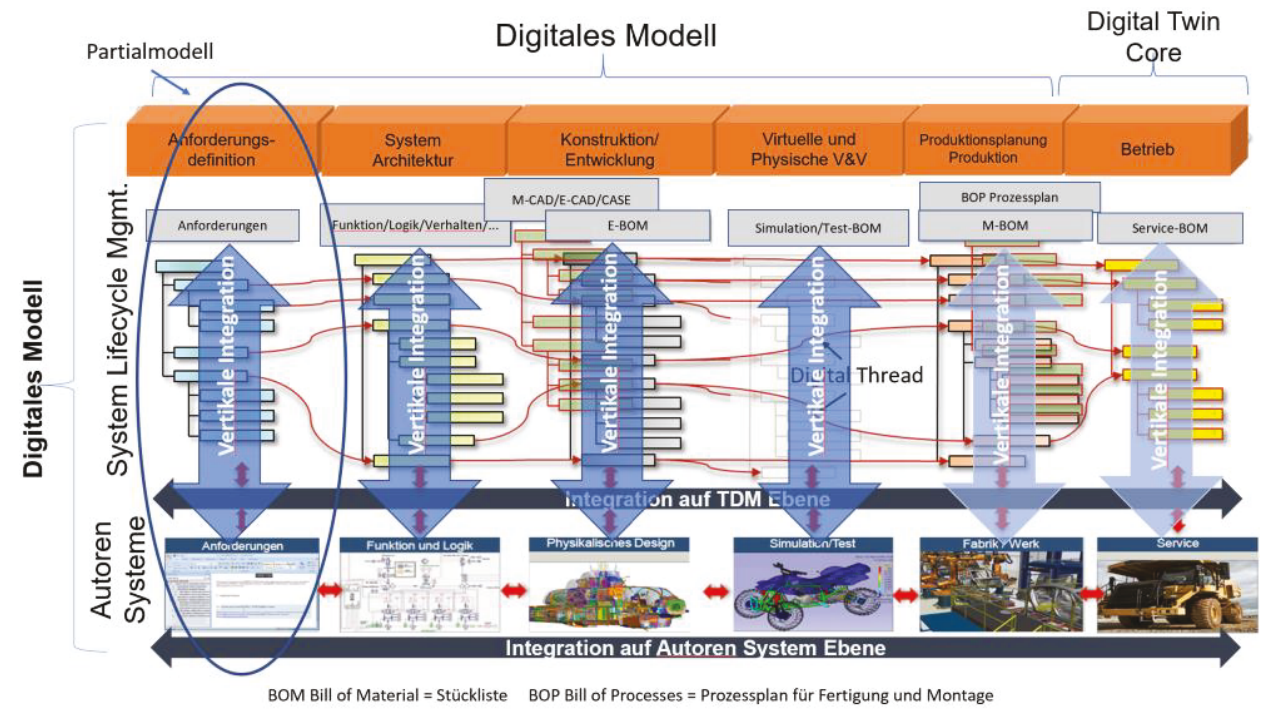

Abbildung 5: Vertikale Integration und Digitalisierung von Autorensystem in TDM und SysLM entlang des Produktlebenszyklus (Eigner, 2021)

Jedes der Partialmodelle bedarf bei der Integration in den SysLM Backbone eines spezifischen Datenmodells. Als ein typisches Beispiel soll hier das Datenmodell der Software-Integration gezeigt werden (Abbildung 6).

Laut einer Untersuchung von Tech-Clarity stellt die bereichsübergreifende Integration des Engineerings für fast die Hälfte der Befragten ein großes Problem dar. So arbeiten die zentralen Entwicklungsbereiche zwar miteinander, aber nicht annähernd genug, um optimale innovative Entwicklungsergebnisse zu erreichen Ohne konsequente Zusammenarbeit und interdisziplinärer Entwicklungsmethodik ist es kaum möglich, innovative Produkte zu entwickeln und dann Änderungen am Produkt und in der Produktion konsequent und qualitätsgerecht umzusetzen. Das Gleiche gilt bei der Nachverfolgung von Schadensfällen. Die Digitalisierungskonzepte Digital Model, Digital Thread und Digital Twin werden in einem SysLM System als Engineering Backbone 
Konzept umgesetzt. Abbildung 7 zeigt das theoretische Optimum einer vollkommenen horizontalen Integration. Die vertikale Integration in den einzelnen Phasen des Produktlebenszyklus muss natürlich implementiert sein und die Partialmodelle existieren.

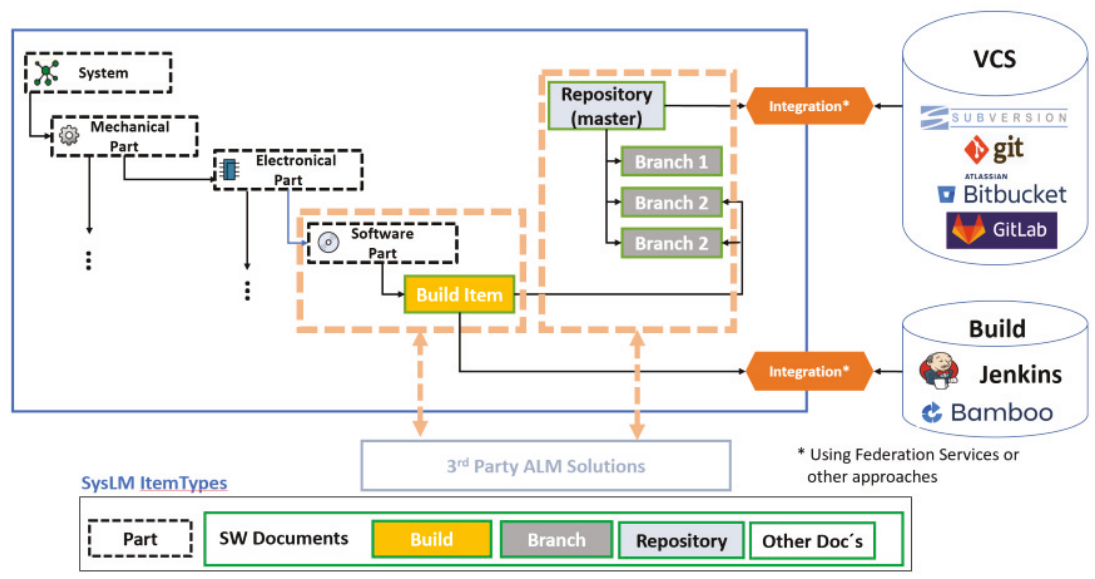

Abbildung 6: Datenmodell für die Software-Integration in SysLM (Eigner, 2021)

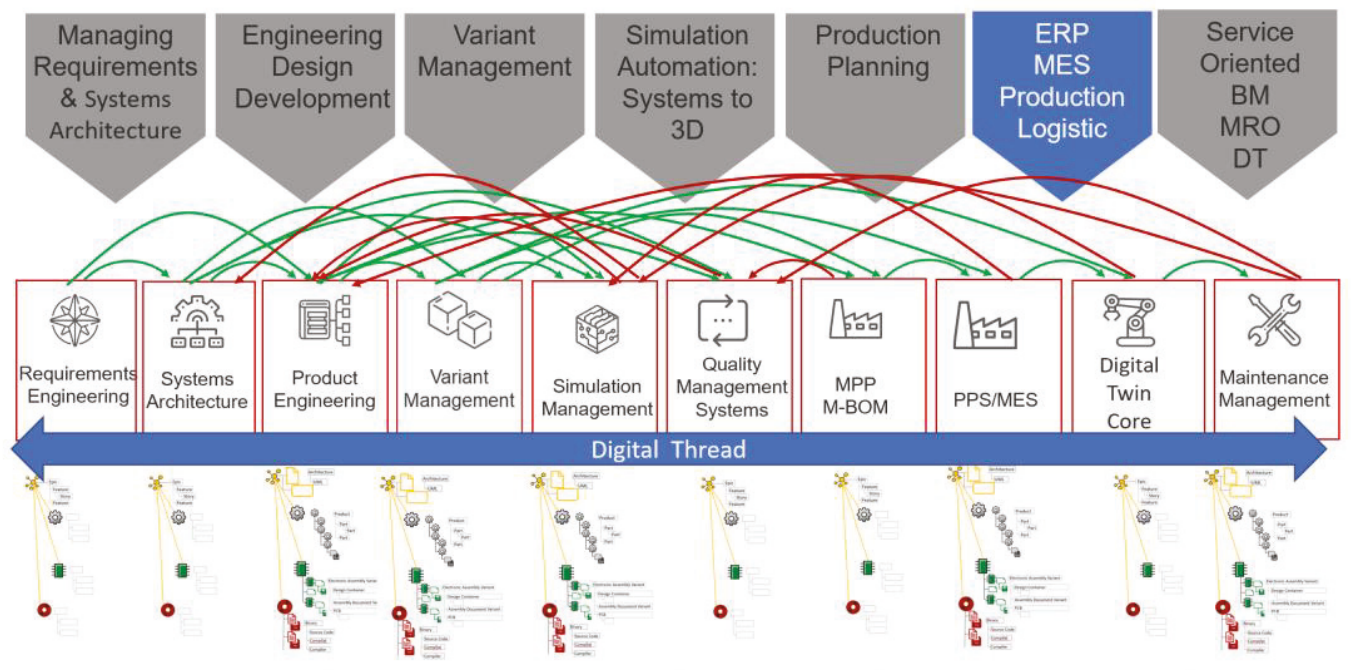

ERP = Enterprise Resource Planning / MES = Manufacturing Execution System / BM = Business Modell / MRO Maintenance Repair Overhaul / DT = Digital Twin

Abbildung 7: Konzept einer Umsetzung einer horizontalen Integration (Eigner, 2021) 
Auch für die horizontale Integration soll ein Beispiel einer partiellen Verknüpfung von Requirements Engineering, System Architektur und Simulation die Komplexität des Datenmodells darstellen (Abbildung 8).

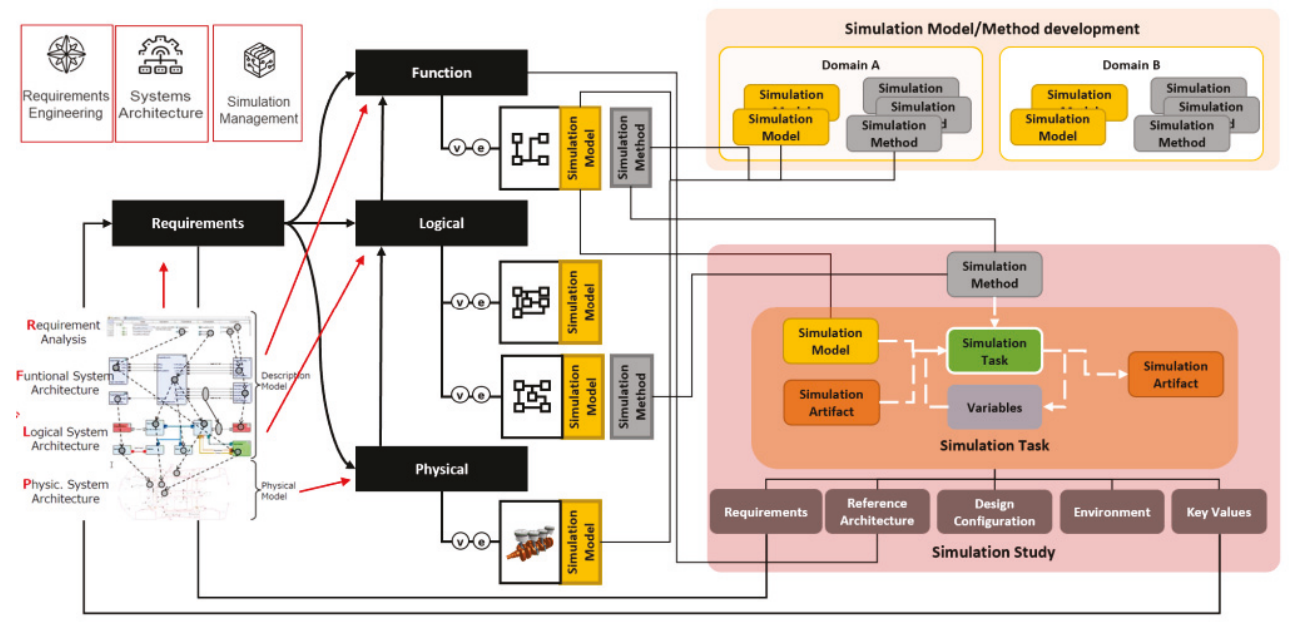

Abbildung 8: Horizontale Integration von Requirements Engineering, System Architektur und Simulation (Chaqdzyński et al 2020)

Keine Firma der Welt hat mit Stand heute eine derartige vollständige horizontale Integration implementiert, sondern nur fragmentarische Ansätze umgesetzt. Aber gerade aus den Erfahrungen dieser Ansätze kann man lernen Die Konzepte liegen vor und erste partielle Prototypen sind aufgebaut worden. Der vor uns liegende Prozess einer Gesamtintegration ist genauso wie der Fortschritt in den letzten 50 Jahren des Einsatzes von Optimierungslösungen im Engineering evolutionär. Die Engineering-relevanten Prozesse, Methoden und IT-Lösungen liegen vor. Sie müssen jetzt auf alle Phasen des Produktlebenszyklus angewandt werden. Das Management muss dahinterstehen und die Mitarbeiter müssen in diesen Change Prozess mitgenommen und integriert werden. 


\section{Literaturverzeichnis}

Ashton, K. (2009), That 'Internet of Things' Thing. Hrsg. RFID Journal Online, https://www.rfidiournal.com/that-internet-ofthings- thing

Broy, M. (2010), Cyber-Physical Systems. Innovation durch software-intensive eingebettete Systeme. Springer, Berlin, Heidelberg

Chądzyński, P.; Panthaki, M.; Nicholich, M.; Asuri, R. (2020), Pervasive Simulation in a PLM Platform - The key to effective management of ever-increasing product complexity, 30 ${ }^{\text {th }}$, Annual Incose, International Symposium, Cape Town, Juli 18-23, 2020

Eigner, M. (2014), "Modellbasierte Virtuelle Produktentwicklung auf einer Plattform für System Lifecycle Management”, in Sendler, U. (Ed.), Industrie 4.0: Beherrschung der industriellen Komplexität mit SysLM, Xpert.press, Springer Vieweg, Berlin, Heidelberg, pp. 91-110.

Eigner, M.; Koch, W.; Muggeo, C. (Eds.) (2017), Modellbasierter Entwicklungsprozess cybertronischer Systeme: Der PLMunterstützte Referenzentwicklungsprozess für Produkte und Produktionssysteme, Springer Vieweg, Berlin

Eigner, M. (2021), System Lifecycle Management - Digitalization of Engineering, Springer Vieweg, Berlin. 10.1007/978-3662-62183-7.

Eigner, M., Dickopf, T. and Apostolov, H. (2019), "Interdisziplinäre Konstruktionsmethoden und -prozesse zur Entwicklung cybertronischer Produkte. Teil 2", In: Konstruktion, 01-02-2019

Estefan, J.A. (2008), "Survey of Model-Based Systems Engineering (MBSE) Methodologies", available at: http://www.omgsysml.org/MBSE_Methodology_Survey_RevB.pdf.

Gill, H.: (2008), A Continuing Vision: Cyber-Physical Systems, Fourth Annual Carnegie Mellon Conference on the Electricity Industry, March 10-11,

Martin, J.N. (1997), Systems engineering guidebook: A process for developing systems and products, Systems engineering series, CRC Press, Boca Raton.

\section{Kontakt}

Prof. Dr.-Ing. Martin Eigner

EIGNER Engineering Consult

Höllhäuserweg 38a

76534 Baden-Baden

Eigner.engineeringconsult@gmail.com 